\title{
APPLIED IMAGISTICS OF ISCHAEMIC HEART A SURVEY. FROM THE EPIDEMIOLOGY OF STABLE ANGINA IN ORDER TO BETTER PREVENT SUDDEN CARDIAC DEATH
}

\author{
RADU EMANUIL PETRUSE \\ Faculty of Engineering/Department IIM., "Lucian Blaga" University of Sibiu, Romania, \\ radu.petruse@ulbsibiu.ro \\ SERGIU BATÂR \\ Faculty of Medicine/ Internal Medicine, "Lucian Blaga" University of Sibiu, Romania, sergiu.batar@yahoo.com
}

ADELA COJAN

Faculty of Medicine/Internal Medicine, "Lucian Blaga" University of Sibiu, Romania, adela_cojan@yahoo.com

IOAN MANITIU

Faculty of Medicine/Department of Public Health and Epidemiology, "Lucian Blaga" University of Sibiu, Romania,ioanmanitiu@yahoo.com

\begin{abstract}
Coronary computed tomography angiography (CCTA) allows coronary artery visualization and the detection of coronary stenoses. In addition; it has been suggested as a novel, noninvasive modality for coronary atherosclerotic plaque detection, characterization, and quantification. Accurate identification of coronary plaques is challenging, especially for the noncalcified plaques, due to many factors such as the small size of coronary arteries, reconstruction artifacts caused by irregular heartbeats, beam hardening, and partial volume averaging. The development of 16, 32, 64 and the latest 320 row multidetector CT not only increases the spatial and the temporal resolution significantly, but also increases the number of images to be interpreted by radiologists substantially. Radiologists have to visually examine each coronary artery for suspicious stenosis using visualization tools such as multiplanar reformatting (MPR) and curved planar reformatting (CPR) provided by the review workstation in clinical practice.
\end{abstract}

Keywords: Ischaemic heart disease, Coronary computed tomography, Automated quantification

\section{Introduction}

Each year, 19 million people worldwide experience a sudden acute coronary event (sudden cardiac death or myocardial infarction). Early detection and accurate assessment of coronary artery disease is crucial in the identification of patients at risk of these highly common yet usually preventable coronary events. Although the current "gold standard" for assessing coronary artery disease is the identification of anatomically significant coronary luminal stenosis by invasive coronary angiography, it is known that most acute coronary syndromes arise from plaques that are not critically occlusive.

Histopathology analyses have shown that the "vulnerable" plaques considered responsible for acute coronary events have a large lipid pool, a thin cap, and macrophage-dense inflammation on or beneath its surface, 4 suggesting that plaque composition and structure may be associated with future coronary events. (Damini Dey, 2009)

2. Prevalence of ischaemic heart disease, stable angina, and sudden cardiac death

According to the current European Guidelines on the management of the stable angina pectoris (Montalescot G 2013), which is used in this moment by the cardiologists across the continent, and is implemented by the European Society of Cardiology through the national societies, the prevalence of angina pectoris is up to 40000 cases per million, varying as first coronary event from 2.6 to 5.4 cases per 1000 persons, each year. These figures actually mean that for instance, a county as Sibiu has around 30 cardiologists which have to deal with around 20000 patients in this moment, only for the issue of ischaemic heart disease. 
In this paper, along with the European guideline we will also often consider data from the American guideline for Diagnosis and Management of patients with stable ischemic heart disease (Stephan D. 2012), which considers data from more recent studies, and is published in December 2012. For the moment, it is important to show that the ischemic disease has a wide spectrum, varying from asymptomatic disease to sudden cardiac death. In this spectrum, the management (including diagnostic and treatment) for acute coronary syndromes is particularly clear about the imagistic strategies and their use. We will study and find populations of patients with stable angina, and those with sudden cardiac death, to determine the necessity of supplemental evaluation of the atherosclerotic plaque, for the prevention of mortality and morbidity in patients where the guidelines currently do not recommend percutaneous coronary interventions or noninvasive assessment of coronary angiography.

Studying the published data, we can see that the current strategies of imagistic evaluation of the coronary plaques limit the use of both non-invasive and invasive techniques in patients with stable coronary disease, by means of cost-efficient strategies, and we will give examples of large studies where the use of Imagistics did not bring real benefits for this population. But on the other hand, these studies show two aspects: first, the current techniques are not completely developed, in order to bring a real benefit in terms of mortality and morbidity, and second, and possibly most important, these studies which constitute the base of guidelines, are carried out in terms of eventual finding stenotic plaques in the coronaries, which can be corrected by invasive therapies, leaving the atherosclerotic non-stenotic soft plaque undiscovered.

For the moment, we refer to the study published by Anselm K. Gitt; Uwe Zeymer; Andrea Papp; Ralf Zahn; Jochen Senges in 2012, in the Journal of the American College of Cardiology, which studied on 2000 patients the effect of interventional therapies (for which, the atherosclerotic plaque should contribute to a stenosis of the vessel by more than $65 \%$ ) where the conclusion is "after 5-year follow-up no differences could be observed between patients with initial interventional versus conservative treatment for stable CAD in clinical practice"( Anselm K. 2012). Still, the mortality in these patients was $17-20 \%$. We believe that concentrating research on these non-stenotic plaques will eventually help both researchers and clinicians in finding strategies to prevent complications of IHD.

Also, there is another sector of population which will eventually benefit from the possibility of non-invasive coronary angiography, the patients with high probability of sudden cardiac death.

In the USA, the incidence of sudden cardiac death is up to 250000 per year. As Chugh SS et al. show in 2008, the previously silent ischaemic coronary disease is likely to be the major cause of SCD -up to $80 \%$ (Sumeet S. 2008)(figure 1), and this statement is powered by research that the same author published years earlier (Chugh SS 2000), where he finds $65 \%$ of the cases with coronary artery disease (figure 3 ).

Figure 1. Coronary disease is responsible for Sudden Cardiac Death, even if it is silent (patients do not have high risk angina prior to death

Etiologies of Sudden Cardiac Death

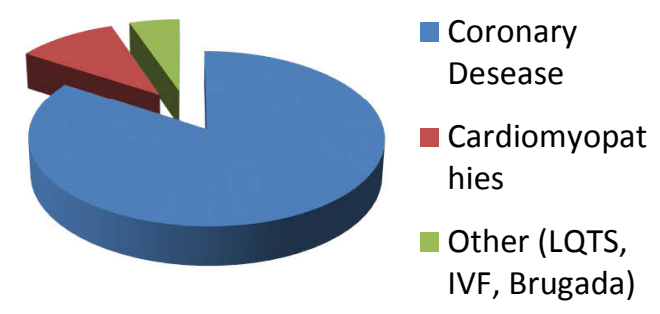

Figure 2. The $33 \%$ of patients having known cardiac disease are a segment of the population which will benefit from better imagistic risk assessment, in order to optimize invasive and drug therapies

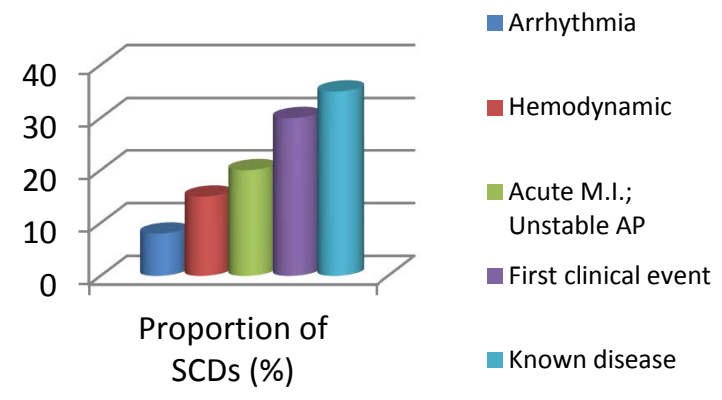

But the spectrum of sudden cardiac death varies: from first clinical event, to acute myocardial infarction, to known disease, but with low individual risk predicted by current strategies (Robert J 2012) (figure 2). 
Myerburg states in 2012 (Robert J. 2012) that broad population profiling has not yet achieved the desired power for individual SCD risk, and SCDs are emerging from subgroups with low risk profiles in large absolute values (figure 2), and there is the need of a more personalized risk evaluation, and the author proposes a set of factors that could be used.

Figure 3. Absolute numbers of Sudden cardiac death in different risk populations reveal the necessity of

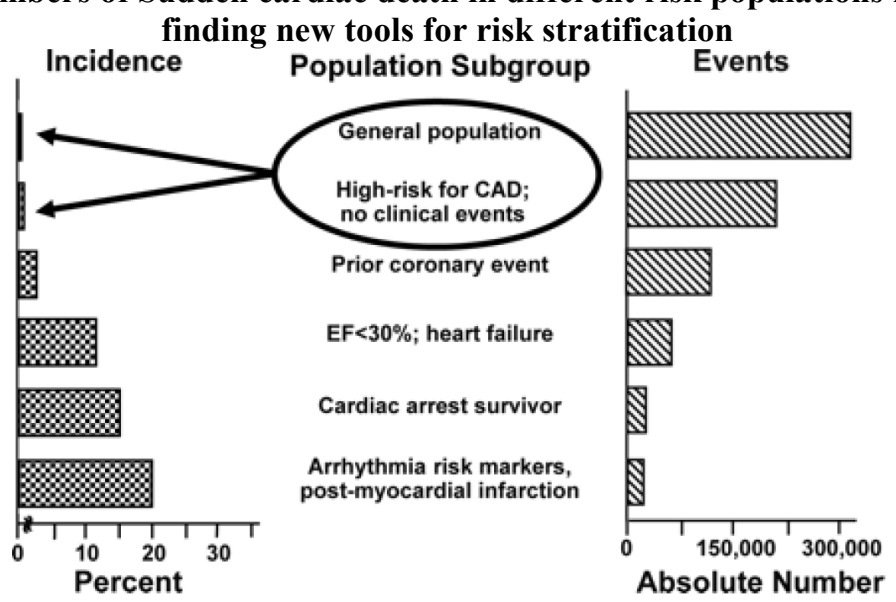

\section{Indications for CTA (computed tomography angiography)}

The current ESC recommendations for CTA are:

Coronary CTA should be considered as an alternative to stress imaging techniques for ruling out SCAD in patients within the lower range of intermediate PTP for SCAD in whom good image quality can be expected (IIa C).

Coronary CTA should be considered in patients within the lower range of intermediate PTP for SCAD after a non conclusive exercise ECG or stress imaging test or who have contraindications to stress testing in order to avoid otherwise necessary invasive coronary angiography if fully diagnostic image quality of coronary CTA can be expected (II a C).

Coronary calcium detection by $\mathrm{CT}$ is not recommended to identify individuals with coronary artery stenosis. (III C)

Coronary CTA is not recommended in patients with prior coronary revascularization. (III C)

Coronary CTA is not recommended as a 'screening' test in asymptomatic individuals without clinical suspicion of coronary artery disease. (III C)

In 2012 Nakanishi et al. publish a study on 134 hypertensive patients, with Multi-detector CT angiography, followed for ACS (acute coronary syndromes) for $39 \pm 10$ months, where they find a positive correlation between low-attenuation plaques, non-obstructive, risk factor, and coronary events, demonstrating the need and capacity of finding a new investigation for preventing acute coronary syndromes, implying the research of non-obstructive coronary plaque (Nakanishi K 2012).

Very recently, the issue whether invasive or medical treatment could be indicated by using non-invasive imaging techniques is studied and published by Schaap $\mathrm{J}$ et al. and they conclude that hybrid myocardial perfusion and CT angiography compared to percutaneous coronary angiography in patients with stable coronary disease has the same results (Schaap J 2013). This is, from our point of view, extremely important most for excluding from coronary invasive procedures the patients who actually do not need stenting, and also making it possible in the same investigation to describe the coronary plaque, not only quantitatively, but also by its intrinsic characteristics.

\section{Detection Methods}

Detection technologies can be roughly split into two categories: intravascular or invasive techniques and non-invasive techniques.

Invasive methods: Intravascular techniques offer a more accurate exam of the plaque. On the other hand, they require a catheter introduction in the patient's vessel. According to the Royal College of Radiologist referral guidelines, Coronary Angiography (CA) is currently the gold standard for diagnosis and evaluation of CAD. However, due to the invasive nature and expense of CA there is a perceived need for a primary non-invasive imaging modality to replace it.

Non-invasive methods: Coronary CT angiography (CCTA) with the use of CT scanners has recently become an increasingly effective clinical tool for non-invasive assessment of the coronary arteries. Recent 
improvements in the high-resolution Multi-Slice Computed Tomography (MSCT) provide information about the arterial wall that is unavailable with most other non-invasive methods, including angiography (Flohr, 2009).

\subsection{Multislice Computed Tomography}

A fundamental advantage of a multislice scanner over monoslice systems is its ability to obtain a first circulation study of a rapidly injected contrast bolus with thinner images. As a result of the shorter acquisition time, the contrast dose can be significantly reduced. Multislice computed tomography (CT) is widely used for assessing coronary atherosclerotic lesions. Several studies reported the use of multislice CT not only for evaluating stenosis severity but also for characterizing coronary plaques and identifying vulnerable lesions, which are prone to plaque rupture and subsequent acute coronary syndrome (ACS). Several CT characteristics of athermanous plaques such as positive remodeling (PR), the presence of adjacent spotty calcification (SP), or the presence of low-density plaque (LDP) are associated with unstable plaques and have been proposed to represent CT markers of plaque vulnerability.

A recently introduced dual-source CT (DSCT) system is equipped with two X-ray tubes and two corresponding detectors. The two acquisition systems are mounted onto the rotating gantry with an angular offset of $90^{\circ}$. The key benefit of DSCT for cardiac scanning is improved temporal resolution

\subsection{Data rates, data transmission and post processing}

With increasing numbers of detector rows and decreasing gantry rotation times, the data transmission systems of MDCT scanners must be capable of handling significant data rates: a four-slice CT system with 0.5 -s rotation time roughly generates $1,000 \times 700 \times 4 \times 2$ bytes $=5.6 \mathrm{MB}$ of data per rotation, corresponding to $11.2 \mathrm{MB} / \mathrm{s}$; a 16-slice CT scanner with the same rotation time generates $45 \mathrm{MB} / \mathrm{s}$, and a 64-slice CT-system can produce up to 180-200 MB/s.

A typical 3D data set is a group of 2D slice images acquired by a CT, MRI, or MicroCT scanner. Usually these are acquired in a regular pattern (e.g., one slice every millimeter) and usually have a regular number of image pixels in a regular pattern. This is an example of a regular volumetric grid, with each volume element, or voxel represented by a single value that is obtained by sampling the immediate area surrounding the voxel.

To render a 2D projection of the 3D data set, one first needs to define a camera in space relative to the volume. Also, one needs to define the opacity and color of every voxel. This is usually defined using an RGBA (for red, green, blue, alpha) transfer function that defines the RGBA value for every possible voxel value.

\section{Atherosclerotic Plaque detection methods using MSCT}

Coronary CTA is typically performed on multidetector CT systems after the injection of iodine contrast media for opacification of the lumen. Coronary arterial plaques are typically reviewed in either axial or multiplanar reformatted planes, as well as in curved multiplanar reformats.

Plaque detection methods can be classified in three major methods:

- Manual - Each image is independently analyzed by a cardiologist or a radiologist

- Semi-automated - Performed using a dedicated software algorithm, the analysis consists by the following automated steps: segmentation of the heart, coronary tree and centerline extraction, and vessel contouring. Subsequently, plaques are manually identified, followed by automatic segmentation, characterization and quantification. Manual corrections to centerline, vessel contours and lesion segmentation can be made by the observer

- Automated-Automatic extraction of the coronary artery trees will reduce the time for coronary analysis. The coronary artery extraction software of the commercial systems is still evolving - some require interactive user-input starting and ending points of the artery of interest, and others attempt to automatically extract the coronary arterial trees without user input. The performance of the commercial software varies from vendor to vendor. For a given vendor, the performance still changes from version to version and the trade-off between sensitivity and specificity varies. However, no vendor software can automatically detect and identify the coronary artery segment with non-calcified plaques and perform MPR or CPR on the coronary artery segment of interest to further speed up coronary analysis. (Chuan Zhou, 2012)

\section{Coronary computed tomography angiography}

The use of CCTA to evaluate plaque characteristics is still subject to controversy. The accuracy of CCTA to identify plaque characteristics using intravascular ultrasound (IVUS) as the gold standard has been reported by several scientists. The comparative studies between CCTA and IVUS have shown that 
partially calcified and calcified lesions can be quite accurately detected by CCTA, whereas non-calcified plaque volume is often underestimated.

Klass O., reported that, in general, demarcating the border of non-calcified plaques is more prone to subjectivity of the reader. Also, an acceptable inter-observer correlation for non-calcified plaques was noted, but higher variability for partially calcified and calcified plaques when a quantification algorithm was used.

Standardized and automated quantification of NCP, CP, and total plaque burden from CCTA, although extremely challenging, is of great interest for refinement of cardiovascular risk stratification. A feasibility study of this approach, have shown that this combined automated algorithm agrees very well with expert manual plaque quantification and is highly reproducible, and the results can be obtained in a fraction of the time $(, 2 \%)$ needed for manual analysis. (Klass O, 2010).

Mark J. Boogers performed a study in 2010 to demonstrate the feasibility of a dedicated algorithm for automated quantification of stenosis severity on multislice computed tomography in comparison with quantitative coronary angiography (QCA). The study was performed on patients who had undergone 64slice computed tomography and invasive coronary angiography, the most severe lesion on QCA was quantified per coronary artery using quantitative coronary computed tomography (QCCTA) software. Additionally, visual grading of stenosis severity using a binary approach was performed. Diameter stenosis (percentage) was obtained from detected lumen contours at the minimal lumen area, and corresponding reference diameter values were obtained from an automatic analysis of the vessel areas within the artery. In this study, a slight underestimation of stenosis severity using QCCTA for noncalcified and mixed plaques was found. Noncalcified and mixed-plaque volumes were significantly underestimated on quantitative MSCT (59.8 $76.6 \mathrm{~mm} 3$ vs. $67.767 .9 \mathrm{~mm} 3$ and $47.787 .5 \mathrm{~mm} 3$ vs. $57.599 .4 \mathrm{~mm} 3$;) compared with IVUSderived plaque volumes, whereas calcified plaques were systematically overestimated $(65.8110 .0 \mathrm{~mm} 3 \mathrm{vs}$. 53.2 90.3 mm3;) on MSCT when compared with IVUS. (Mark J. Boogers, 2010)

Another study has been done by Stella-Lida Papadopoulou in 2011, when she evaluated the ability of 64-slice multidetector computed tomography (MDCT)-derived plaque parameters to detect and quantify coronary atherosclerosis, using intravascular ultrasound (IVUS) as the reference standard.

The analysis on a segmental basis revealed that MDCT correctly detected 213 of 220 segments with any atherosclerotic plaque (sensitivity $96 \%$ ), whereas the presence of any plaque was correctly ruled out in 28 of 32 segments (specificity $88 \%$ ). The majority of the segments with plaque detected by MDCT contained non-calcified plaque (154 out of $213,72 \%)$. Mixed plaque was present in 44 segments $(21 \%)$ and calcified plaque in 15 segments $(7 \%)$. Four nondiseased segments were incorrectly characterized as containing non-calcified plaque. The present study demonstrated that 64-MDCT is able to detect and quantify atherosclerotic plaque using MDCT-derived parameters. Further improvement in CT resolution is required for more reliable assessment of very small and distal coronary plaques. (Stella-Lida Papadopoulou, 2011)

Ki-Woon Kang performed a study to demonstrate the feasibility of an auto-CAD algorithm for cCTA in patients presenting with acute chest pain. 398 consecutive patients were investigated (229 male, mean age $50 \pm 21$ years) who had acute chest pain and underwent cCTA. All cCTA data were analyzed using an auto-CAD algorithm for the detection of $>50 \%$ CAD on cCTA. The accuracy of auto-CAD was compared with the formal radiology report. In 380 of 398 patients (18 were excluded due to failure of data processing), per-patient analysis of auto-CAD revealed the following: sensitivity $94 \%$, specificity $63 \%$, positive predictive value (PPV) 76\%, and negative predictive value (NPV) 89\%. . (Ki-Woon Kang, 2012)With further improvements in hardware and software, contrast enhanced coronary CTA may become part of the armamentarium in the quest for the detection of the "vulnerable plaque" and the "vulnerable patient" so that appropriate preventive measures can be instituted in a targeted fashion, at least partially based on the findings of coronary CTA. (Szilard Voros, 2011)

\section{Conclusions}

For the population of patients with stable ischaemic heart disease, classified as low-risk patients, supplemental methods for studying coronary atherosclerosis, and implementing these in everyday practice, would have real benefits in terms of morbidity, mortality, and cost-efficiency for long term therapy management.

Accurate detection of coronary atherosclerotic plaques by CT remains difficult but can be performed with modern equipment, after careful patient selection and with sufficient expertise. Attempts at plaque quantification and characterization have been successful, but further refinements regarding reproducibility, accuracy, and ability to predict future events are required. 
With recent developments in image acquisition and reconstruction technologies, coronary CTA can be performed with relatively low radiation exposure. With further technological innovation and clinical research, coronary CTA may become an important tool in the quest to identify vulnerable plaques and the at-risk patient.

\section{References}

- Anselm K. Gitt; Uwe Zeymer; Andrea Papp; Ralf Zahn; Jochen Senges. Impact of interventional versus conservative approach on 5 year mortality of patients with stable angina and documented coronary artery disease in clinical practice: results of the STAR registry. J Am Coll Cardiol. 2012;59(13s1):E1378-E1378.

- Chuan Zhou, H.-P. C. (2012). Automated coronary artery tree extraction in coronary CT angiography using a multiscale enhancement and dynamic balloon tracking (MSCAR-DBT) method. Computerized Medical Imaging and Graphics .

- Chugh SS, Kelly KL, Titus JL. Sudden cardiac death with apparently normal heart. Circulation. 2000 Aug 8;102(6):649-54.

- D. Dey, V.Y. Cheng, P.J. Slomka, R. Nakazato, A. Ramesh, S. Gurudevan, G. Germano, D.S.: Automated 3-dimensional quantification of noncalcified and calcified coronary plaque from coronary CT angiography, Journal of cardiovascular computed tomography 3 (6), 372-382

- $\quad$ Flohr T.G., Leng S., Yu L., Aiimendinger T., Bruder H., Petersilka M., Eusemann C.D., Stierstorfer K., Schmidt B., McCollough C.H.: Dual-source spiral CT with pitch up to 3.2 and $75 \mathrm{~ms}$ temporal resolution: image reconstruction and assessment of image quality, Med Phys. 2009, Dec; 36(12) 5641-53.

- Klass O, K. S. (2010). Coronary plaque imaging with 256-slice multidetector computed tomography: interobserver variability of volumetric lesion parameters with semiautomatic plaque analysis software. Int $\mathrm{J}$ Cardiovasc Imaging .

- Mark J. Boogers, J. D. (2010). Automated Quantification of Stenosis Severity on 64-Slice CT. Cardiovascular Imaging .

- Montalescot G. et al. 2013 ESC guidelines on the management of stable coronary artery disease The Task Force on the Management of Stable Angina Pectoris of the European Society of Cardiology. Eur Heart J European Heart Journal (2013) 34, 2949-3003

- Nakanishi K, Fukuda S, Shimada K, Ehara S, Inanami H, Matsumoto K, Taguchi H, Muro T, Yoshikawa J, Yoshiyama M. Non-obstructive low attenuation coronary plaque predicts three-year acute coronary syndrome events in patients with hypertension: multidetector computed tomographic study. J Cardiol. 2012 Mar;59(2):167-75

- Robert J. Myerburg; M. Juhani Junttila. Sudden Cardiac Death Caused by Coronary Heart Disease. Circulation. 2012; 125: 1043-1052

- Schaap J, de Groot JA, Nieman K, Meijboom WB, Boekholdt SM, Post MC, Van der Heyden JA, de Kroon TL, Rensing BJ, Moons KG, Verzijlbergen JF. Hybrid myocardial perfusion SPECT/CT coronary angiography and invasive coronary angiography in patients with stable angina pectoris lead to similar treatment decisions. Heart. $2013 \mathrm{Feb}$;99(3):188-94

- Stella-Lida Papadopoulou, L. A.-L. (2011). Detection and quantification of coronary atherosclerotic plaque by 64-slice multidetector CT: A systematic head-to-head comparison with intravascular ultrasound. In Atherosclerosis (pp. 163-170). Elsevier.

- Stephan D. Fihn et al. 2012 ACCF/AHA/ACP/AATS/PCNA/SCAI/STS Guideline for the Diagnosis and Management of Patients With Stable Ischemic Heart Disease: Executive Summary. J Am Coll Cardiol. 2012;60(24):2564-2603

- Sumeet S. Chugh, Kyndaron Reinier, Carmen Teodorescu, Audrey Evanado, Elizabeth Kehr, Mershed Al Samara, Ronald Mariani, Karen Gunson, Jonathan Jui. Epidemiology of Sudden Cardiac Death: Clinical and Research Implications. Prog Cardiovasc Dis. 2008; 51(3): 213-228.

- Szilard Voros, S. R. (2011). Coronary Atherosclerosis Imaging by Coronary CT Angiography. The American College of Cardiology Foundation . 